\title{
Top pair production at NNLO
}

\author{
Sebastian Sapeta*t \\ Institute of Nuclear Physics, Polish Academy of Sciences \\ ul. Radzikowskiego 152, 31-342 Kraków, Poland \\ E-mail: sebastian.sapeta@ifj.edu.pl
}

\begin{abstract}
We report on progress towards calculating the NNLO cross section for top quark pair production in the the framework which combines the $q_{T}$-slicing method and small- $q_{T}$ factorization. In this contribution, we present the complete result for the small- $q_{T}$ soft function at NNLO, which constitutes the main challenge on the way to our goal. In order to evaluate the divergent integrals appearing in the calculation, we develop methods based on sector decomposition and differential equations. We present complete validation of our framework and provide results which are ready for application in the calculation of the $t \bar{t}$ cross section at NNLO.
\end{abstract}

Loops and Legs in Quantum Field Theory (LL2018)

29 April 2018 - 04 May 2018

St. Goar, Germany

\footnotetext{
* Speaker.

${ }^{\dagger}$ Based on work done in collaboration with Michał Czakon and René Ángeles-Martínez.
} 


\section{Introduction}

The increasing accuracy of experimental data coming from the LHC is in many cases superior to that of theoretical predictions. If it comes to corrections from Quantum Chromodynamics, the state of the art for most processes of interest has recently changed from NLO to NNLO. Some gaps still need to be filled, however, and additional work is required to fully test the NNLO results and implement them in efficient public codes such that they can benefit experimental analyses. At the same time, the calculation of the $\mathrm{N}^{3} \mathrm{LO}$ cross section for the Higgs boson production from gluon fusion [1] has marked the first step on the way towards pushing the accuracy of QCD predictions to the next level.

One of the most important class of measurements studied at the LHC are processes which involve production of the top quark. And, in particular, top-anti-top production, which is relevant both in studies of properties of the Standard Model, as well as in searches for new physics, as it forms significant backgrounds to many signatures. The cross section for this process is currently known up to NNLO [2, 3, 4, 5, 6, 7].

In this proceedings, we present the calculation of the complete, small- $q_{T}$ NNLO soft function for top quark pair production. This result, together with the framework and tools developed to obtain it, form key elements of an alternative calculation of the complete NNLO cross section for that process.

The motivation behind our work is two-fold. On one hand, it will lead to a second, independent result for the NNLO top pair production cross section, which, given the complexity of the calculation, is highly desirable. At the same time, our work forms a stepping stone towards developing a general framework for calculations of $\mathrm{N}^{3} \mathrm{LO} \mathrm{QCD}$ corrections to a wide range of processes of relevance for hadron colliders.

\section{Theoretical framework}

The approach for achieving NNLO accuracy for the top pair production, presented in this proceedings, is part of a wider strategy for calculating $\mathrm{N}^{m} \mathrm{LO}$ contributions to processes of the type

$$
h_{1}+h_{2} \rightarrow F\left(q_{T}\right)+X
$$

where two hadrons, $h_{1}$ and $h_{2}$, collide and produce an object $F$, which is registered in a detector, together with an undetected QCD radiation $X$. Our framework is suitable both when $F$, whose transverse momentum is denoted by $q_{T}$, is a colour-neutral object (single EW boson, pair of EW bosons, the Higgs), as well as when it carries colour, like in the case of the top quark pair.

If we choose to use the $q_{T}$-slicing method, we are able to write the cross section as a sum of two components $[8,9]$

$$
\frac{\sigma_{\mathrm{N}^{m} \mathrm{LO}}^{F}}{d \Phi}=\int_{0}^{q_{T \mathrm{cut}}} d q_{T} \frac{d \sigma_{\mathrm{N}^{m} \mathrm{LO}}^{F}}{d \Phi d q_{T}}+\int_{q_{T \mathrm{cut}}}^{\infty} d q_{T} \frac{d \sigma_{\mathrm{N}^{m-1} \mathrm{LO}}^{F+\text { jet }}}{d \Phi d q_{T}}
$$

each of which is separately finite. The advantage of this approach is that the second term in the above equation, which represents resolved emissions, is required only at the $\mathrm{N}^{m-1} \mathrm{LO}$ accuracy, and it is already known in most relevant cases. On the contrary, the first term in Eq. (2.2), which 
combines virtual and unresolved real corrections, is usually unknown. However it is needed only in the small- $q_{T}$ approximation.

In order to calculate the latter we use the Soft Collinear Effective Theory (SCET) [10], in which the cross section factorizes at small $q_{T}$ according to the formula

$$
\frac{d \sigma_{\mathrm{N}^{m} \mathrm{LO}}^{F}}{d \Phi d q_{T}}=\mathcal{B}_{1}^{\mathrm{N}^{m} \mathrm{LO}} \otimes \mathcal{B}_{2}^{\mathrm{N}^{m} \mathrm{LO}} \otimes \mathcal{H}^{\mathrm{N}^{m} \mathrm{LO}} \otimes \mathcal{S}^{\mathrm{N}^{m} \mathrm{LO}}+\mathcal{O}\left(\frac{q_{T}^{2}}{q^{2}}\right)
$$

where $q^{2}$ is the invariant mass of the object $F$. The functions appearing in the above equation account for contributions coming from different phase space regions of $X$. Specifically, the beam functions $\mathcal{B}_{i}$ sum up emissions of collinear and anti-collinear partons, the hard functions, $\mathcal{H}$, accounts for hard radiation, and the soft function, $\mathcal{S}$, sums emissions from soft, real gluons. The calculation of each of these functions is considerably simpler than the calculation of the complete cross section. In addition, some of the functions are already available from the literature.

In the case of the top quark pair production, the beam functions are known up to NNLO [11, 12] and the NNLO hard function can be extracted from Refs. [13, 14]. However, the small- $q_{T}$ soft function is only known up to NLO $[15,16]$. Hence, in order to achieve the NNLO accuracy of the $t \bar{t}$ cross section, one needs to calculate the missing NNLO correction to the soft function appearing in Eq. (2.3) (We note that such calculation shares many features with that of the NNLO soft function for top pair production in the threshold limit $[17,18]$. However, the result for the latter is not of direct use in our context.)

The soft function for our process of interest can be schematically defined with the following equation

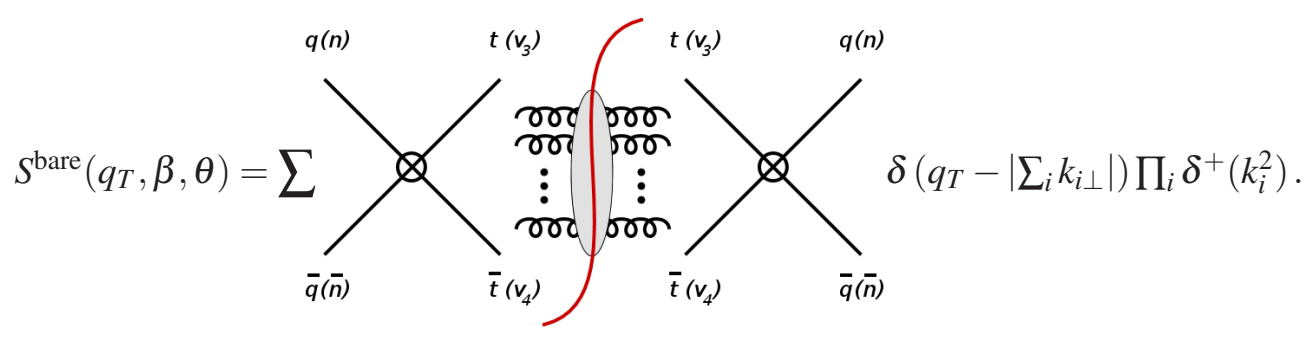

To this end, we focus on the $q \bar{q} \rightarrow t \bar{t}$ subprocess and introduce the following notation for the 4momenta: $p_{q}=m_{t} n, p_{\bar{q}}=m_{t} \bar{n}$ and $p_{t}=m_{t} v_{3}+l_{3}, p_{\bar{t}}=m_{t} v_{4}+l_{4}$, where $n=(1,0,0,1), \bar{n}=$ $(1,0,0,-1)$. We see that in the definition (2.4), the transverse momenta of real emissions are restricted to sum up to a fixed value of $q_{T}$. Because the gluons are soft, $l_{i} \ll m_{t} v_{i}$, and the velocities satisfy Born kinematics, $n+\bar{n}=v_{3}+v_{4}$, at each perturbative order. Apart from $q_{T}$, the soft function of Eq. (2.4) depends on $\beta=\sqrt{1-4 m_{t}^{2} / q^{2}}$ and $\theta$, where the latter is the scattering angle of the top quark in the $t \bar{t}$ rest frame.

The NNLO soft function corresponds to a sum of all $\mathcal{O}\left(\alpha_{s}^{2}\right)$ contributions from Eq. (2.4). They take forms of $2 d$-dimensional integrals which exhibit soft and rapidity singularities. The latter arise when the light-cone components of the gluon 4-momenta become very small or very large, and are not removed by dimensional regularization. In our calculation, we adopt the prescription of Ref. [19] which turns the above divergences into poles in a new regulator $\alpha$. Even though the individual integrals suffer from rapidity divergences, their complete sum, hence the soft function, is finite in the limit $\alpha \rightarrow 0[15,19]$. 
Each function defined on the right hand side of Eq. (2.3), when calculated directly from diagrammatic definitions, like the one given in Eq. (2.4) for the soft function, is separately divergent. These divergencies correspond to soft and collinear limits and they must cancel between the hard, soft and beam functions, as the entire cross section has to be finite.

It turns out to be useful to remove divergences also at the level of the functions entering the factorization formula (2.3). This can be achieved by the procedure of multiplicative renormalization. For example, the renormalized soft function is given by

$$
\boldsymbol{S}(\mu)=\boldsymbol{Z}_{S}^{\dagger}(\mu, \varepsilon) \boldsymbol{S}^{\mathrm{bare}}(\varepsilon) \boldsymbol{Z}_{s}(\mu, \varepsilon),
$$

where the coefficient $\boldsymbol{Z}_{s}(\mu, \varepsilon)$ absorbs all soft divergences such that $\boldsymbol{S}(\boldsymbol{\mu})$ is finite.

As usual in the procedure of renormalization, the renormalized object acquires dependence on an arbitrary parameter $\mu$, which has to vanish at the level of the cross section. This implies that the renormalized soft function of small $-q_{T}$ factorization must satisfy the following RGE equation [20]

$$
\frac{d}{d \ln \mu} \boldsymbol{S}_{i \bar{i}}(\mu)=-\boldsymbol{\gamma}_{i \bar{i}}^{s \dagger} \boldsymbol{S}_{i \bar{i}}(\mu)-\boldsymbol{S}_{i \bar{i}}(\mu) \boldsymbol{\gamma}_{i \bar{i}}^{s},
$$

where

$$
\boldsymbol{\gamma}_{i \bar{i}}^{s}=\boldsymbol{\gamma}_{i \bar{i}}^{h}-2 \gamma^{i} \mathbf{1}
$$

and $\boldsymbol{\gamma}_{i \bar{i}}^{h}$ is defined as a non- $\Gamma_{\text {cusp }}$ part of the full anomalous dimension matrix $\Gamma$ [21], while $\gamma^{i}$ is the massless-particle anomalous dimension (and enters RGE equations for beam functions in DrellYan and Higgs production [22,23]). The soft anomalous dimension matrix $\boldsymbol{\gamma}^{s}$ is related to the soft renormalization factor (also a matrix in colour space), $\boldsymbol{Z}_{s}$, as follows

$$
\boldsymbol{\gamma}^{s}=-\boldsymbol{Z}_{s}^{-1} \frac{d \boldsymbol{Z}_{s}}{d \ln \mu} .
$$

Each quantity in Eq. (2.5) has a perturbative expansion in the strong coupling $\alpha_{s}$. This allows one to relate the bare and renormalized objects order by order. At NNLO, the renormalized soft function is given by

$$
\boldsymbol{S}_{\mathrm{ren}}^{(2)}=\boldsymbol{Z}_{s}^{\dagger(2)} \boldsymbol{S}_{\text {bare }}^{(0)}+\boldsymbol{S}_{\text {bare }}^{(0)} \boldsymbol{Z}_{s}^{(2)}+\boldsymbol{Z}_{s}^{\dagger(1)} \boldsymbol{S}_{\text {bare }}^{(0)} \boldsymbol{Z}_{s}^{(1)}+\boldsymbol{Z}_{s}^{\dagger(1)} \boldsymbol{S}_{\text {bare }}^{(1)}+\boldsymbol{S}_{\text {bare }}^{(1)} \boldsymbol{Z}_{s}^{(1)}+\boldsymbol{S}_{\text {bare }}^{(2)}-\frac{\beta_{0}}{\varepsilon} \boldsymbol{S}_{\text {bare }}^{(1)} .
$$

The quantity on the left hand side is finite, while the objects on the right hand side exhibit divergencies in the soft limit. These divergencies are dimensionally regularized (in the $\overline{\mathrm{MS}}$ scheme) and they correspond to poles in $\varepsilon$. The first three terms on the right hand side of Eq. (2.9) contain the pole part only, while other terms have both finite and pole parts. The poles of the bare soft function, $\boldsymbol{S}_{\text {bare }}^{(2)}$, which we obtain through direct calculation following the definition of Eq. (2.4), should match the poles from the other terms on the right hand side of Eq. (2.9), which can be determined from the renormalization group. The cancellation of $\varepsilon$ poles provides a strong cross check which we will use as part of the validation of our framework.

\section{NNLO soft function: methods of calculation}

The soft function at NNLO receives contributions from several classes of diagrams. The complete formula can be schematically written as

$$
S_{\text {bare }}^{(2)}=S_{2 \text {-cut, } q \bar{q}}+S_{2 \text {-cut, } g g}+S_{1 \text {-cut }}+S_{0 \text {-cut }} .
$$



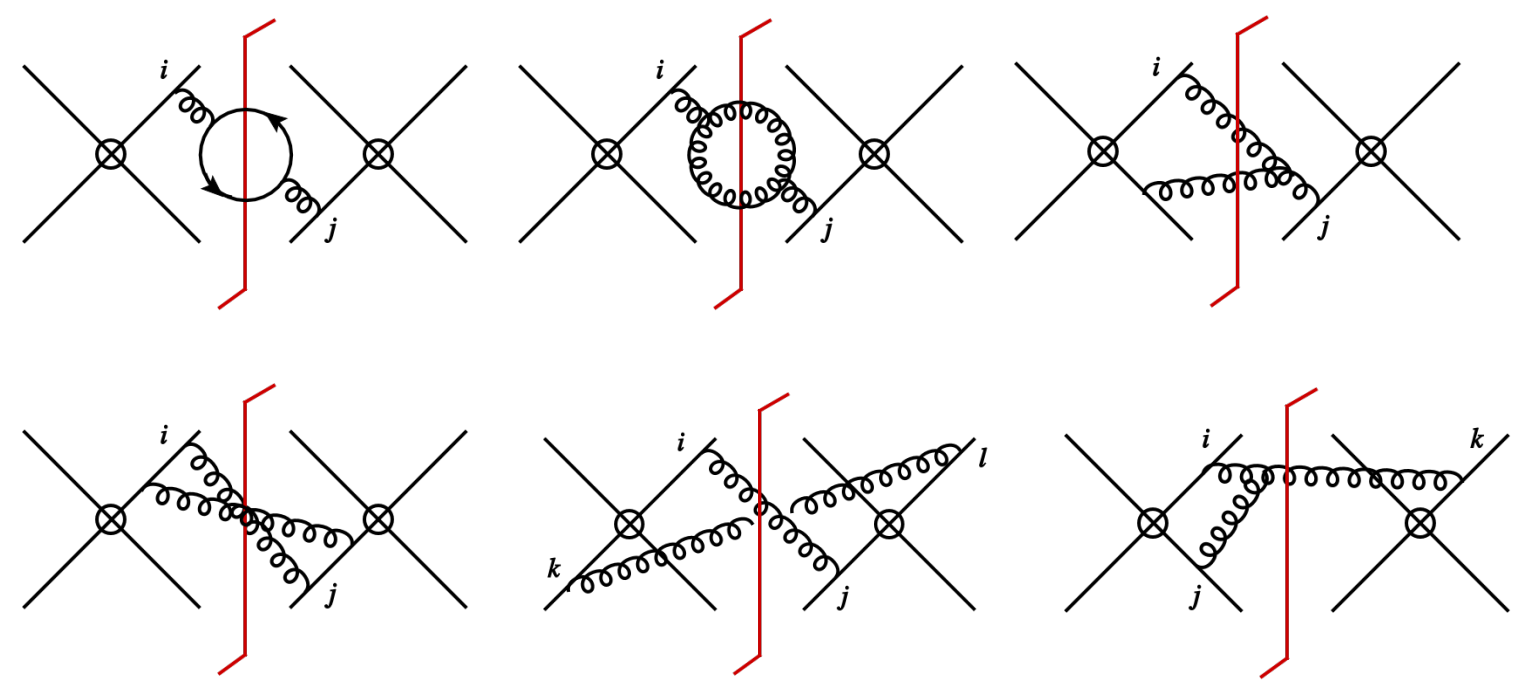

Figure 1: Example diagrams contributing to the NNLO soft function for top pair production.

Example diagrams are given in Fig. 1. They include quark bubble, gluon bubble, abelian and nonabelian graphs. We also notice that gluons can connect different numbers of distinct Wilson lines: two, three or four.

We apply different methods of calculation to different classes of integrals. The bubble diagrams, as well as parts of the non-abelian, three-Wilson line, double-cut graphs, which we call "tadpole", are calculated almost entirely analytically with help of the method of differential equations. Single-cut integrals are calculated directly by a combination of analytic and numerical methods. The remaining integrals, namely those coming from double-cut, non-tadpole diagrams, are computed with the method of sector decomposition, adapted to our specific problem. This set of integrals poses the most difficult challenge in our calculation.

With an exception of the propagators introduced to regularize rapidity divergences, the bubble diagrams depend only on the momenta $k$ and $k+l$. This feature allows one to first integrate them over $k+l$, a generalization of the standard vacuum polarization tensor calculation, and then solve the integral over $k$ with the method of differential equations.

Since the gluons connected to the Wilson lines are not cut in the bubble diagrams, the integrals involve a function of $\theta\left(k^{2}\right)$. In our calculation we trade it for the Dirac delta function at the cost of introducing an extra integral over the mass by applying the identity $1=\int_{0}^{\infty} d m^{2} \delta\left(k^{2}-m^{2}\right)$. This allows us then to use reverse unitarity and turn all delta functions into propagators. We obtain a topology which consists of six propagators. All the integrals needed for the bubble diagrams can be reduced to five master integrals for which we then derive a set of differential equations with respect to the variable $\beta$. For reduction, we used our private $\mathrm{C}++$ implementation of the Laporta algorithm [24], which depends on FORM [25] and Fermat [26]. The structure of the set differential equations is such that the general solutions for masters can be obtained iteratively as a series in $\alpha$ and $\varepsilon$.

To calculate the double-cut contributions to the NNLO soft function, we designed the following integration strategy. We start from analytically integrating 3 out of $2 d$ dimensions. Then, 
we map the remaining momenta into a unit hypercube (splitting the integral if necessary) and apply sector decomposition $[27,28]$ to disentangle overlapping singularities. Finally, we expand the result in $\alpha$ and $\varepsilon$ and numerically integrate the coefficients with help of the CUBA library [29].

\section{NNLO soft function: results}

The bare NNLO soft function has the following structure

$$
S_{\text {bare }}^{(2)}\left(L_{\perp}, \beta, \theta\right)=\frac{1}{\varepsilon^{2}} S^{(2,-2)}\left(L_{\perp}\right)+\frac{1}{\varepsilon} S^{(2,-1)}\left(L_{\perp}\right)+S^{(2,0)}\left(L_{\perp}\right)
$$

where $L_{\perp}=\ln \left(x_{T}^{2} \mu^{2} / 4 e^{-2 \gamma_{E}}\right)$ and $x_{T}$ is a coordinate-space variable related to $q_{T}$ through Fourier transform.

All the pole terms, as well as the $L_{\perp}$-dependent part of the finite term in Eq. (4.1) can be predicted from the renormalization group. The only contributions that has to be obtained through direct calculation is the $L_{\perp}$-independent part of $S^{(2,0)}\left(L_{\perp}\right)$. Nevertheless, we calculate all terms, singular, finite, $L_{\perp}$-dependent and $L_{\perp}$-independent, and use the redundant ones for cross checks against the renormalization group predictions.

Even though the NNLO soft function exhibits at most $1 / \varepsilon^{2}$ singularity, higher-order $\varepsilon$ poles, as well as $\alpha$ poles, appear in contributions from individual diagrams. We checked that all $\alpha$ poles, including $\varepsilon / \alpha$, as well as the $1 / \varepsilon^{4}$ pole cancel within each colour structure. For example, the coefficient in front of $1 / \varepsilon^{4}$ in the complete NNLO soft function for the $q \bar{q}$ channel reads

$$
\left(\begin{array}{cc}
0.003 N_{c}-0.003 N_{c}{ }^{-1} & 0.003 N_{c}^{-2}+0.0008 N_{c}^{2}-0.004 \\
0.003 N_{c}^{-2}+0.0008 N_{c}^{2}-0.004 & -0.002 N_{c}^{-3}+0.003 N_{c}^{-1}+0.0005 N_{c}^{3}-0.001 N_{c}
\end{array}\right),
$$

where we used some specific values of $\beta=0.4$ and $\theta=0.5$. We see that the above result is compatible with zero. We note that only double cut diagrams contribute to this pole.

Unlike the $1 / \varepsilon^{4}$, and all the $\alpha$ poles, the $1 / \varepsilon^{3}$ pole does not cancel within individual contributions defined in Eq. (3.1). It cancels however between the 1-cut and 2-cut parts whose sum reads

$$
\left(\begin{array}{cc}
0.0002 N_{c}^{-1}-0.0001 N_{c}^{3}-0.0001 N_{c} & -0.0003 N_{c}^{-2}+0.000 N_{c}^{2}+0.0001 \\
-0.0003 N_{c}^{-2}+0.0002 N_{c}^{2}+0.0001 & 0.0002 N_{c}^{-3}-0.0001 N_{c}^{-1}-0.0003 N_{c}^{3}+0.0001 N_{c}
\end{array}\right),
$$

where, again, we used the values of $\beta=0.4$ and $\theta=0.5$.

The double and single poles do not vanish, but, as mentioned earlier, they have to match predictions from the RGE. And indeed, we see that

$$
\begin{aligned}
& {\left[S_{\text {direct }}^{(2,-2)}+S_{\mathrm{RGE}}^{(2,-2)}\right]_{\beta=0.4, \theta=0.5}=} \\
& \quad\left(\begin{array}{cc}
-0.008 N_{c}^{-1}-0.00006 N_{c}^{3}+0.008 N_{c} & 0.007 N_{c}^{-2}+0.001 N_{c}^{2}-0.009 \\
0.007 N_{c}^{-2}+0.001 N_{c}^{2}-0.009 & -0.005 N_{c}^{-3}+0.007 N_{c}^{-1}+0.0005 N_{c}^{3}-0.002 N_{c}
\end{array}\right),
\end{aligned}
$$



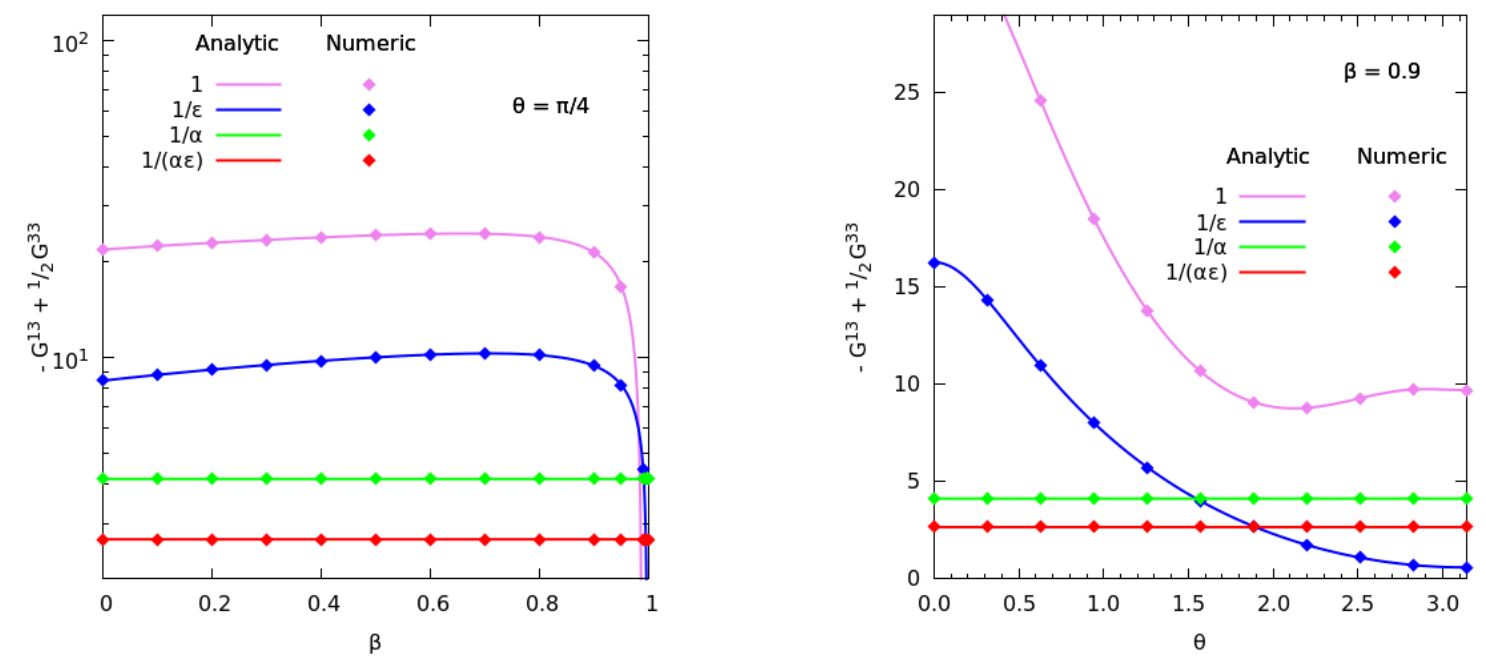

Figure 2: Comparison of numeric and analytic results for example graphs contributing to the $n_{f}$ part of the NNLO soft function.

for the double pole, and

$$
\begin{aligned}
& {\left[S_{\text {direct }}^{(2,-1)}+S_{\mathrm{RGE}}^{(2,-1)}\right]_{\beta=0.4, \theta=0.5}=} \\
& \quad\left(\begin{array}{cc}
0.002 N_{c}^{-1}-0.0003 N_{c}^{3}-0.002 N_{c} & -0.002 N_{c}^{-2}-0.0008 N_{c}^{2}+0.003 \\
-0.002 N_{c}^{-2}-0.0008 N_{c}^{2}+0.003 & 0.002 N_{c}^{-3}-0.003 N_{c}^{-1}-0.0001 N_{c}^{3}+0.001 N_{c}
\end{array}\right),
\end{aligned}
$$

for the single pole.

The results from Eqs. (4.4) and (4.5) correspond to the real part of the NNLO soft function. At this order, the soft function contains also an imaginary part, which originates from single-cut diagrams. We have checked that our direct calculation perfectly reproduces the imaginary part of the coefficients of the $1 / \varepsilon^{2}$ and $1 / \varepsilon$ poles predicted by renormalization group.

The above results provide strong checks of our framework. We were, however, able to validate it further by comparing the numerical results for combinations of quark-bubble graphs, obtained with the sector decomposition-based method, and the analytic results from the method of differential equations. An example comparison is presented in Fig. 2 where the points (lines) correspond to numeric (analytic) coefficients of different powers in the expansion in $\alpha$ and $\varepsilon$. We observe per-mille-level agreement for this and all the remaining graphs that contribute to the $n_{f}$ part of the NNLO soft function.

Having done all the validations and cross checks, we are now in a position to calculate the finite, $L_{\perp}$-independent part of the bare NNLO soft function. By combining it with appropriate contributions coming from $\boldsymbol{S}_{\text {bare }}^{(1)}$, following Eq. (2.9), we obtain the renormalized soft function, which, in the $q \bar{q}$ channel and for a specific phase-space point, reads

$$
S_{q \bar{q}}^{(2) \text { ren }}\left(\beta=\frac{2}{5}, \theta=\frac{\pi}{4}\right)=\left(\begin{array}{cc}
198.682 & -8.73644 \\
-8.73644 & 211.739
\end{array}\right)+i\left(\begin{array}{cc}
0 & 23.0178 \\
23.0178 & 0
\end{array}\right) .
$$

For more results, we refer the reader to Ref. [30]. 


\section{Conclusions}

We presented the calculation of the complete, small- $q_{T}$ soft function for top pair production at NNLO. In order to evaluate the most difficult divergent integrals, originating from non-bubble, double cut diagrams, we developed a framework based on sector decomposition. Other integrals were calculated either directly, like in the case of single-cut diagrams, or by the method of differential equations, which was applied to bubble integrals. We performed a three-step validation of our results: by verifying that rapidity singularities, and higher-order $\varepsilon$ poles cancel, by cross-checking that our direct calculation reproduces all terms predicted by the renormalization group and, finally, by finding a perfect agreement between numeric results from the sector decomposition-based framework and analytic results, for the graphs involving gauge, ghost or quark bubble. The NNLO, small- $q_{T}$ soft function can now be used to obtain full $t \bar{t}$ cross section at NNLO by means of the $q_{T}$-slicing method, as well as for small- $q_{T}$ resummation at NNLL'.

\section{Acknowledgements}

This work has been supported by the National Science Centre, Poland grant POLONEZ No. 2015/19/P/ST2/03007. The project has received funding from the European Union's Horizon 2020 research and innovation programme under the Marie Skłodowska-Curie grant agreement No. 665778. We are grateful to Mateusz Dobija for implementation of numerical integration with CUBA.

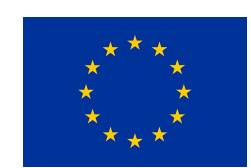

\section{References}

[1] C. Anastasiou, C. Duhr, F. Dulat, F. Herzog and B. Mistlberger, Phys. Rev. Lett. 114 (2015) 212001 doi:10.1103/PhysRevLett.114.212001 [arXiv:1503.06056 [hep-ph]].

[2] P. Bärnreuther, M. Czakon and A. Mitov, Phys. Rev. Lett. 109 (2012) 132001 doi:10.1103/PhysRevLett.109.132001 [arXiv:1204.5201 [hep-ph]].

[3] M. Czakon and A. Mitov, JHEP 1301 (2013) 080 doi:10.1007/JHEP01(2013)080 [arXiv:1210.6832 [hep-ph]].

[4] M. Czakon and A. Mitov, JHEP 1212 (2012) 054 doi:10.1007/JHEP12(2012)054 [arXiv:1207.0236 [hep-ph]].

[5] M. Czakon, P. Fiedler and A. Mitov, Phys. Rev. Lett. 110 (2013) 252004 doi:10.1103/PhysRevLett.110.252004 [arXiv:1303.6254 [hep-ph]].

[6] M. Czakon, D. Heymes and A. Mitov, Phys. Rev. Lett. 116 (2016) no.8, 082003 doi:10.1103/PhysRevLett.116.082003 [arXiv:1511.00549 [hep-ph]].

[7] M. Czakon, P. Fiedler, D. Heymes and A. Mitov, JHEP 1605 (2016) 034 doi:10.1007/JHEP05(2016)034 [arXiv:1601.05375 [hep-ph]].

[8] S. Catani and M. Grazzini, Phys. Rev. Lett. 98 (2007) 222002 doi:10.1103/PhysRevLett.98.222002 [hep-ph/0703012].

[9] R. Bonciani, S. Catani, M. Grazzini, H. Sargsyan and A. Torre, Eur. Phys. J. C 75 (2015) no.12, 581 doi:10.1140/epjc/s10052-015-3793-y [arXiv:1508.03585 [hep-ph]]. 
[10] T. Becher, A. Broggio and A. Ferroglia, Lect. Notes Phys. 896 (2015) pp.1 doi:10.1007/978-3-319-14848-9 [arXiv:1410.1892 [hep-ph]].

[11] T. Gehrmann, T. Lubbert and L. L. Yang, Phys. Rev. Lett. 109 (2012) 242003 doi:10.1103/PhysRevLett.109.242003 [arXiv:1209.0682 [hep-ph]].

[12] T. Gehrmann, T. Luebbert and L. L. Yang, JHEP 1406 (2014) 155 doi:10.1007/JHEP06(2014)155 [arXiv:1403.6451 [hep-ph]].

[13] M. Czakon, Phys. Lett. B 664 (2008) 307 doi:10.1016/j.physletb.2008.05.028 [arXiv:0803.1400 [hep-ph]].

[14] P. Bärnreuther, M. Czakon and P. Fiedler, JHEP 1402 (2014) 078 doi:10.1007/JHEP02(2014)078 [arXiv:1312.6279 [hep-ph]].

[15] H. T. Li, C. S. Li, D. Y. Shao, L. L. Yang and H. X. Zhu, Phys. Rev. D 88 (2013) 074004 doi:10.1103/PhysRevD.88.074004 [arXiv:1307.2464 [hep-ph]].

[16] S. Catani, M. Grazzini and A. Torre, Nucl. Phys. B 890 (2014) 518 doi:10.1016/j.nuclphysb.2014.11.019 [arXiv:1408.4564 [hep-ph]].

[17] A. Ferroglia, B. D. Pecjak, L. L. Yang, B. D. Pecjak and L. L. Yang, JHEP 1210 (2012) 180 doi:10.1007/JHEP10(2012)180 [arXiv:1207.4798 [hep-ph]].

[18] G. Wang, X. Xu, L. L. Yang and H. X. Zhu, JHEP 1806 (2018) 013 doi:10.1007/JHEP06(2018)013 [arXiv:1804.05218 [hep-ph]].

[19] T. Becher and G. Bell, Phys. Lett. B 713 (2012) 41 doi:10.1016/j.physletb.2012.05.016 [arXiv:1112.3907 [hep-ph]].

[20] H. X. Zhu, C. S. Li, H. T. Li, D. Y. Shao and L. L. Yang, Phys. Rev. Lett. 110 (2013) no.8, 082001 doi:10.1103/PhysRevLett.110.082001 [arXiv:1208.5774 [hep-ph]].

[21] V. Ahrens, A. Ferroglia, M. Neubert, B. D. Pecjak and L. L. Yang, JHEP 1009 (2010) 097 doi:10.1007/JHEP09(2010)097 [arXiv:1003.5827 [hep-ph]].

[22] T. Becher and M. Neubert, Eur. Phys. J. C 71 (2011) 1665 doi:10.1140/epjc/s10052-011-1665-7 [arXiv:1007.4005 [hep-ph]].

[23] T. Becher, M. Neubert and D. Wilhelm, JHEP 1305 (2013) 110 doi:10.1007/JHEP05(2013)110 [arXiv:1212.2621 [hep-ph]].

[24] S. Laporta, Int. J. Mod. Phys. A 15 (2000) 5087 doi:10.1016/S0217-751X(00)00215-7, 10.1142/S0217751X00002157 [hep-ph/0102033].

[25] B. Ruijl, T. Ueda and J. Vermaseren, arXiv:1707.06453 [hep-ph].

[26] R. H. Lewis, Computer Algebra System Fermat, http://home.bway.net/lewis .

[27] T. Binoth and G. Heinrich, Nucl. Phys. B 585 (2000) 741 doi:10.1016/S0550-3213(00)00429-6 [hep-ph/0004013].

[28] T. Binoth and G. Heinrich, Nucl. Phys. B 680 (2004) 375 doi:10.1016/j.nuclphysb.2003.12.023 [hep-ph/0305234].

[29] T. Hahn, Comput. Phys. Commun. 168 (2005) 78 doi:10.1016/j.cpc.2005.01.010 [hep-ph/0404043].

[30] R. Ángeles-Martínez, M. Czakon, S. Sapeta, to appear. 